\title{
Identification of Metallurgical Surface Finish Images-In Manufacturing Process using Fuzzy Classifier
}

\author{
Shailendra M.Mukane,Ph.D \\ Department of Electronics and \\ Telecommunication Engg. \\ SVERI's College of Engineering \\ Pandharpur, Solapur Maharashtra
}

\author{
Feiroz F. Shaikh \\ Department of Electronics Engg. \\ Walchand Institute of \\ Technology, Solapur \\ Maharashtra.
}

\begin{abstract}
Surface metrology with image processing is a challenging task having wide application in the industry. Analysis of surface finish can be done using image processing. The proposed system aims at identifying three classes of surface finish images, viz. Casting, Milling and shaping. The system proposes an effective combination of features for analysis of the engineering surfaces. The prototype system developed for classification of surface finish images using Discrete Wavelet Transform (DWT) and Fuzzy Logic classifier. The feature extracted using DWT are Standard Deviation and Mean. The system out performs the earlier methods and gives $92.78 \%$ of average success rate for only 72 number of features. The system is also analysed by different wavelet filters for maximum success rate and minimum success rate comparison.
\end{abstract}

\section{General Terms}

Surface finish identification of metallurgical images, Texture Analysis and Fuzzy logic

\section{Keywords}

Image processing, Discrete Wavelet Transform, Wavelet Statistical features, fuzzy classifier.

\section{INTRODUCTION}

The need of quality control and performance testing has become a vital part of the production procedure. Surface finish plays an important role in several engineering applications. That's the reason the process of inspection needs be computerized automated ([1], [2]) and inspection results should be fed back to the upstream manufacturing processes for improvement of quality of product. The inspection system should be designed to be an efficient composition of human cleverness and experience along with the fortress of a machine.

Several methods for surface inspection problems were designed during the recent years. Researchers have exposed that the automated machine vision has the advantage of being non-contact than the contact methods [3]. The intensity histogram of surface image is utilized to characterize surface roughness and quality of imaes [4]. Irem Y. Tumer et al. [5] explains four methods yielding mathematical measures to examine the precision of parts of manufactured surfaces. The eigenvalues of the covariance matrix helps to calculate average energy. S.Livens et al. [6] explains intuitive properties like roughness, granulation and regularity. Features which reflect scale-dependent properties can be extracted from each sub image separately. Wong et al. [7] has used the co-occurrence matrix and the fuzzy logic theorem to detect the casting surface defects. By applying Discrete Wavelet Transform under different light conditions, surface roughness features for metal machining, grinding and polishing operation were extracted [8]. W. Zeng et al. [9] has stated that
DT-CWT filter is very suitable for the separation and extraction of the frequency components such as surface roughness, waviness and form. Smriti H. Bhandari et al. [10] proposed a method that analyzes the surface with discrete wavelet transform (DWT) and dual-tree complex wavelet transform (DT-CWT). Standard Deviation, Kurtosis and Canny edge detector features are being considered. Canberra distance metric were used for comparison between the surface class's viz. Casting, Milling and Shaping.

Four types of classification methods such as parametric, nonparametric, stochastic methods, non-metric methods are there which were enlighten by R.O. Duda [11]. Classification process involves classifying images based on the feature vectors of query image and feature database provided by the feature extraction methods. From Bayesian decision theory classification methods start. If no prior parameterized knowledge about the probability structure then classification is based on nonparametric technique. Various types of classification techniques are there which are based on information provided by training samples alone. These techniques are fuzzy classification, neural network approach, etc. Engine Avci [12] proposed multilayer perceptron neural network classifier to classify selected texture images. I.Turkoglu et al. [13] presented a comparison of wavelet support vector machine (W-SVM) and wavelet-adaptive network based fuzzy inference system (W-ANFIS) approaches for texture image classification. Both W-SVM and W-ANFIS methods are used for classification of the 22 texture images. [14]

In this paper, a combination of two texture features namely Standard Deviation and Energy distribution is considered. DWT with different wavelet functions have been used as tools for the analysis of the system. Fuzzy classifier is used for correct classification of images.

\section{DISCRETE WAVELET TRANSFORM}

In case of DWT, a time-scale representation of the digital signal is obtained using digital filtering technique. The signal to be analysed is passed through filters with different cut-off frequencies at different scales.

DWT is computed by successive lowpass and highpass filtering of the discrete time-domain signal as shown in Fig. 1. This is called the Mallat algorithm or Mallat-tree decomposition. Its significance is in the manner it connects the continuous time mutiresolution to discrete-time filters. In figure, the signal is denoted by the sequence $x[n]$, where $n$ is an integer. The lowpass, filter is denoted by $\mathrm{G}_{0}$ while the high pass filter is denoted by $\mathrm{H}_{0}$. At each level, the high pass filter produces detail information $d[n]$, while the low pass filter associated with scaling function produces coarse approximations $\mathrm{a}[\mathrm{n}]$. 


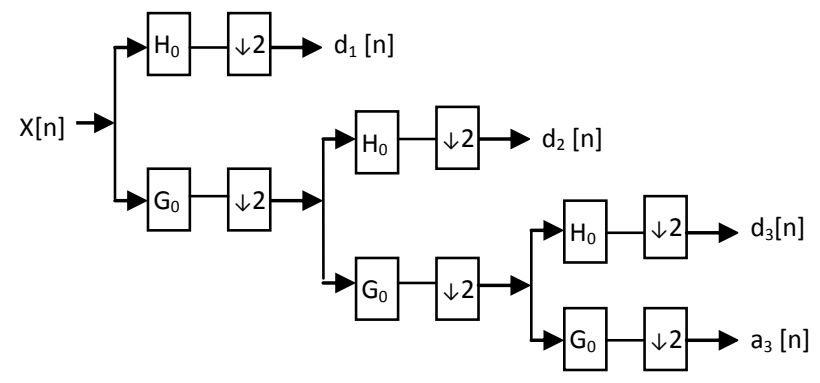

Figure 1: Three-level wavelet decomposition tree

At each decomposition level, the half band filters produce signals spanning only half the frequency band. This doubles the frequency resolution as the uncertainty in frequency is reduced by half. In accordance with Nyquist's rule if the original signal has a highest frequency of $\omega$, which requires a sampling frequency of $2 \omega$ radians, then it now has a highest frequency of $\omega / 2$ radians. It can now be sampled at a frequency of $\omega$ radians thus discarding half the samples with no loss of information. This decimation by 2 halves the time resolution as the entire signal is now represented by only half the number of samples. Thus, while the half band low pass filtering removes half of the frequencies and thus halves the resolution the decimation by 2 doubles the scale.

With this approach, the time resolution becomes arbitrarily good at high frequencies, while the frequency resolution become arbitrarily good at low frequencies. The filtering and decimation process is continued until the desired level is reached. The maximum number of levels depends on the length of the signal. The DWT of the original signal is then obtained by concatenating all the coefficients, $a[n]$ and $d[n]$, starting from the last level of decomposition.

\section{WAVELET STATISTICAL FEATURES}

The wavelet transform is best suitable for multi-resolution approach. It decomposes a signal with a family of basis functions obtain through conversion and dilation of a mother wavelet. When DWT is applied to image, it decomposes the image into four sub-bands.

Form the extracted wavelet coefficients, Energy (1) and standard deviation (2) of all the sub-bands up to 5 and 9 level of decomposition are calculated by using the equations

$$
\begin{aligned}
E_{k} & =\frac{1}{N^{2}} \sum_{i=1}^{N} \sum_{J=1}^{N}\left|x_{k}(i, j)\right| \\
\sigma_{k} & =\left[\frac{1}{N^{2}} \sum_{i=1}^{N} \sum_{J=1}^{N}\left(x_{k}(i, j)-\mu_{k}(i, j)\right)^{2}\right]^{\frac{1}{2}}
\end{aligned}
$$

where $E_{k}$ is the energy $\& \sigma_{K}$ be the standard deviation for the Kth sub-band of dimension $\mathrm{NxN}$ and coefficients are $x_{K}(i, j) \&$ mean value is $\mu_{K}(i, j)$ [14].

For different texture images, above features are computed for training and testing and stored in feature vectors as Wavelet Statistical Features (WSF).

\section{THE PROPOSED SYSTEM}

Three classes of images viz. casting, milling and shaping are considered. The purpose of the study is to classify images with respective to the classes to which they belongs and try to improve the results as compared to existing methods [10]. The proposed system has three major stages as shown in Fig. 2

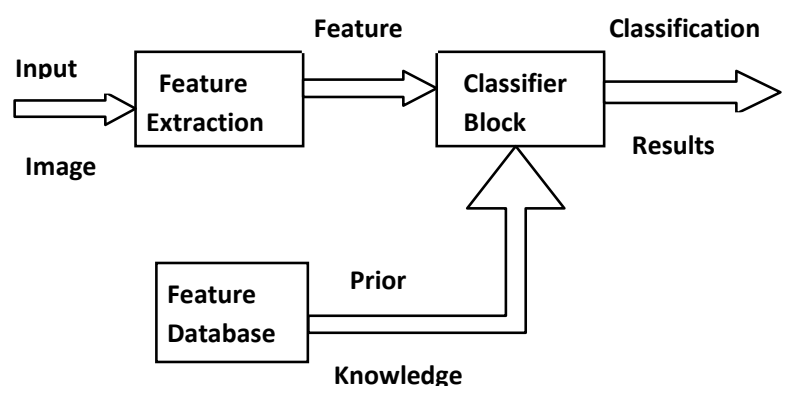

Figure 2: A Typical Feature Extraction \& Classification Scheme

\section{- Feature Extraction}

Different class surface finish images are taken as input query image. By applying DWT, expected features extracted and stored as feature vector for testing.

\section{- Feature Database}

The other samples of surface finish images are taken as standard features for comparison and stored as feature database.

\section{- Classifier Block}

The algorithm designed to classify the input images with standard database. Here fuzzy logic based classifier is used for classification.

\section{FUZZY LOGIC CLASSIFIER}

Fuzzy logic can be a form of many-valued logic or probabilistic logic; it works on the basis of reasoning that is approximate rather than fixed and exact. In this system various kinds of knowledge can be represented in terms of fuzzy set also it can model the interactions and relationships among the system variables. Fuzzy logic deals with innovative tools to handle the complex and ill-defined systems where classical tools become unsuccessful. Fuzzy systems are universal approximators of non liner functions. Two aspects are important in fuzzy system one generating the best rule set and second tuning the membership functions. These should relate properly the independent and dependent variable.

The Inputs to the fuzzy system are wavelet statistical features that are covered in this research. The outputs of the system are performance of the rule.

Fuzzy sets with Gaussian membership functions are designed for the classification of input variables. These fuzzy sets can be defined using the following equation [15].

$$
\mu(f)=e^{-0.5\left(\frac{f-m}{\sigma}\right)^{2}}
$$

where ' $m$ ' is the mean of the fuzzy set and ' $\sigma$ ' is the standard deviation from the mean.

Rules for the fuzzy system are obtained by fuzification of the numerical values from wavelet. The fuzzy sets corresponding to each texture feature are generated and maximum degree of membership will be 1 for each fuzzy set. The standard deviation of feature values from the mean is calculated. Each texture feature is assigned to the fuzzy set with the maximum 
degree of membership. Success rate is calculated using the results obtained after deffuzification [14].

\section{EXPERIMENTAL RESULTS AND DISCUSSION}

The experiment is carried out on three texture databases. The databases are prepared by taking images of the standard (master) roughness comparison specimen manufactured by three machining processes viz. milling, casting and shaping.

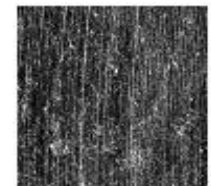

$6 s$

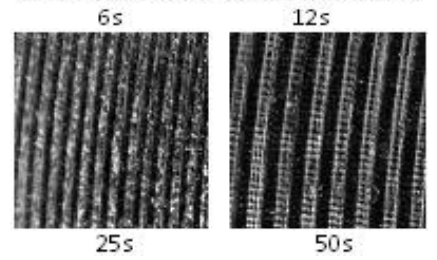

Figure 3: Casting Database [10]
(Only flat i.e. non-curved surfaces are used.). Four sub classes viz 6, 12, 25 and 50 seconds images from each database milling ,casting and shaping have been taken.

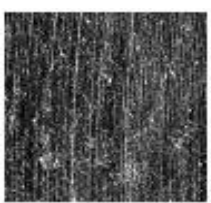

$6 s$

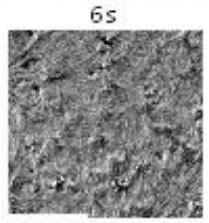

$25 \mathrm{~s}$

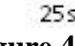

Figure 4: Milling Database [10]

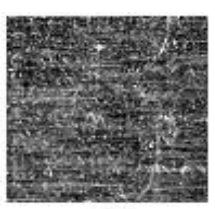

$6 s$

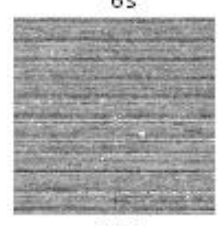

$25 s$

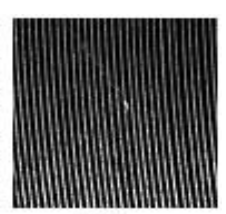

$12 \mathrm{~s}$

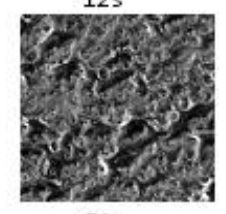

$50 \mathrm{~s}$

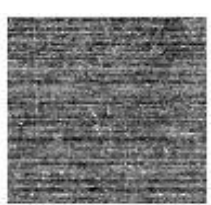

$12 \mathrm{~s}$

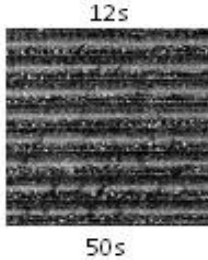

$50 \mathrm{~s}$
Figure 5: Shaping Database [10]

One image from each class in the database can be seen in Fig. 3 5. Label associated with an image indicates no. of seconds of machining process having some surface roughness value. Each class is having 120 gray scale images. Hence sixty from each class are used for training purpose where as remaining sixty are tested for classification. Images of size 256 X 256 pixels are used. Ratio of training to testing is maintained 1:1. In this experiment pyramid structured type of DWT is used with different wavelet filter.
One feature database is created using wavelet decomposed sub-bands up to $5^{\text {th }}$ as well as for $9^{\text {th }}$ level of decomposition. Total number of sub-bands up to fifth level will be 20 and for $9^{\text {th }}$ level will be 36.Energy (1) and standard deviations (2) of each sub-band coefficients are calculated for each level and 60 number of samples. These features are stored as Wavelet Statistical Features (WSF). In this way maximum WSF for milling and shaping and casting will (20x2) 40 and (36x2)72.

1) WSF1- Energy only [20 features].

2) WSF2- Energy + Standard Deviation [40 features].

3) WSF3- Energy + Standard Deviation [72 features].

The comparative chart is shown in table. 1

Table 1. Classification Performance with WSF1

\begin{tabular}{|c|c|c|c|}
\hline \multirow{2}{*}{ TD } & \multicolumn{3}{|c|}{ WSF1 } \\
\cline { 2 - 4 } MW & Casting & \multicolumn{1}{c|}{ Milling } & Shaping \\
\hline db2 & 20 & 98.33 & 55 \\
\hline coif1 & 18.33 & 98.33 & 58.33 \\
\hline Haar & 18.33 & 98.33 & 65 \\
\hline Bior1.1 & 18.33 & 98.33 & 65 \\
\hline sym2 & 20 & 98.33 & 55 \\
\hline dmey & 23.33 & 98.33 & 53.33 \\
\hline
\end{tabular}

Table 2. Classification Performance with WSF2

\begin{tabular}{|c|c|c|c|}
\hline \multirow{2}{*}{ TD } & \multicolumn{3}{|c|}{ WSF2 } \\
\cline { 2 - 4 } MW & Casting & Milling & Shaping \\
\hline db2 & 68.33 & 100 & 98.33 \\
\hline coif1 & 70 & 100 & 98.33 \\
\hline Haar & 51.67 & 98.33 & 100 \\
\hline Bior1.1 & 51.67 & 98.33 & 100 \\
\hline sym2 & 68.33 & 100 & 98.33 \\
\hline dmey & 68.33 & 98.33 & 96.67 \\
\hline
\end{tabular}

Table 3. Classification Performance with WSF3

\begin{tabular}{|c|c|c|c|}
\hline \multirow{2}{*}{ TD } & \multicolumn{3}{|c|}{ WSF3 } \\
\cline { 2 - 4 } MW & Casting & Milling & Shaping \\
\hline db2 & 100 & 100 & 98.33 \\
\hline coif1 & 71.67 & 100 & 98.33 \\
\hline Haar & 68.33 & 98.33 & 100 \\
\hline Bior1.1 & 71.67 & 98.33 & 100 \\
\hline sym2 & 100 & 100 & 98.33 \\
\hline dmey & 75 & 98.33 & 96.67 \\
\hline \multicolumn{4}{|c}{}
\end{tabular}

TD - Texture Descriptor db2 - Daubechies

MW- Mother Wavelet Bior1-Boiorthogonal coif1-Coiflet sym2-Symlet 


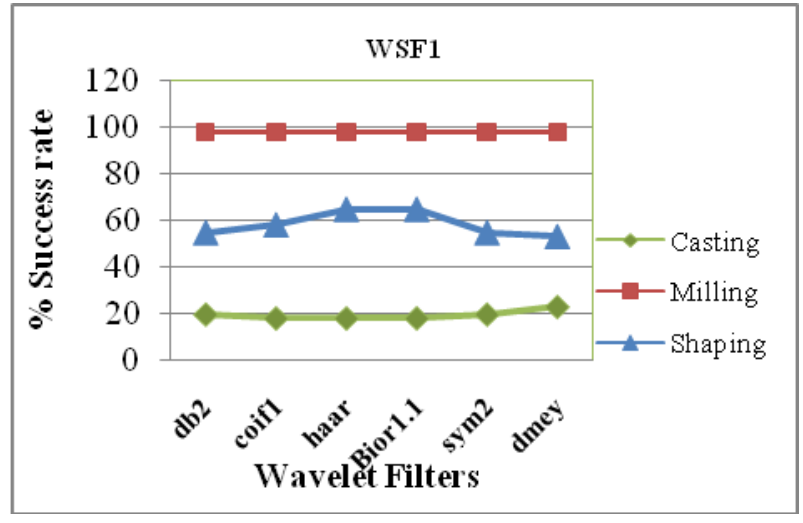

Figure 6: Maximum success rate with WSF1

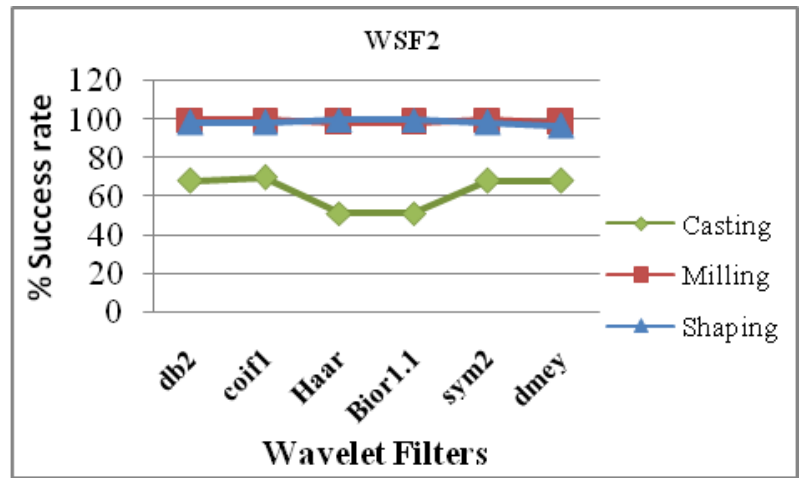

Figure 7: Maximum success rate with WSF2

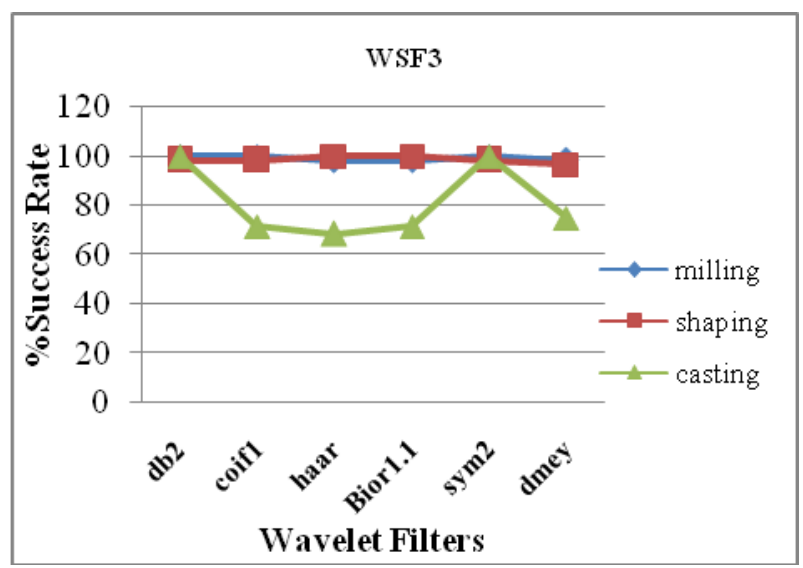

Figure 7: Maximum success rate with WSF3

In terms of success rate, performance of the above feature sets is tested with the help of a fuzzy classifier. Let $\mathrm{N}_{\mathrm{T}}$ be the no. of samples to be tested and out of that if the system correctly classifies $N_{C}$ times then success rate of the system for the fuzzy rule as a percentage is given by Eq.4 [16].

$$
\mathrm{S}_{\mathrm{R}}=\mathrm{Nc} / \mathrm{N}_{\mathrm{T}} \times 100
$$

By observation of Fig. 6 and Fig. 7 it is noticed that for WSF1 result is satisfactory only for milling which offers $98.33 \%$ upto 5 level of decomposition, WSF2 offers $99.16 \%$ and $98.61 \%$ success rate for milling and shaping respectively. While WSF3 offers $81.11 \%$ for casting upto 9 level of decomposition [14].
The results of this research work are compared with the earlier work published in the literature as presented in the table 4 as

Table 4. Comparison of Proposed method with Earlier published method[10].

\begin{tabular}{lcc}
\hline $\begin{array}{l}\text { Texture classification } \\
\text { Method from literature }\end{array}$ & $\begin{array}{c}\text { Average Correct } \\
\text { Classification }\end{array}$ & No of Features \\
\hline $\begin{array}{l}\text { DT-CWT \& } \\
\text { Canny edge detector }\end{array}$ & 91.27 & 147 \\
& & \\
DWT-proposed method & 92.78 & 72 \\
\hline
\end{tabular}

The results of Proposed method and the results of Earlier published method shows that average success rate for proposed system is somewhat improved for minimum number of features that earlier method. Hence proposed system is much better than earlier for correct classification.

Table 5. Maximum success rate comparison of proposed method with Earlier published method [10].

\begin{tabular}{|c|c|c|c|}
\hline Method type & Milling & Casting & Shaping \\
\hline $\begin{array}{l}\text { DWT-Propose } \\
\text { Method }\end{array}$ & 100 & 100 & 100 \\
\hline $\begin{array}{c}\text { DT-CWT\& } \\
\text { Canny edge } \\
\text { detector[10] }\end{array}$ & 95.56 & 84.07 & 94.17 \\
\hline
\end{tabular}

The table 5.11 of comparison with earlier methods reveals that for few wavelet functions the success rate for milling, casting and shaping will be $100 \%$ for proposed system where as for Bhandhari and Deshpande method it hardly reaches to $95 \%$. For casting it is less than $90 \%$ as shown in Figure 5.16

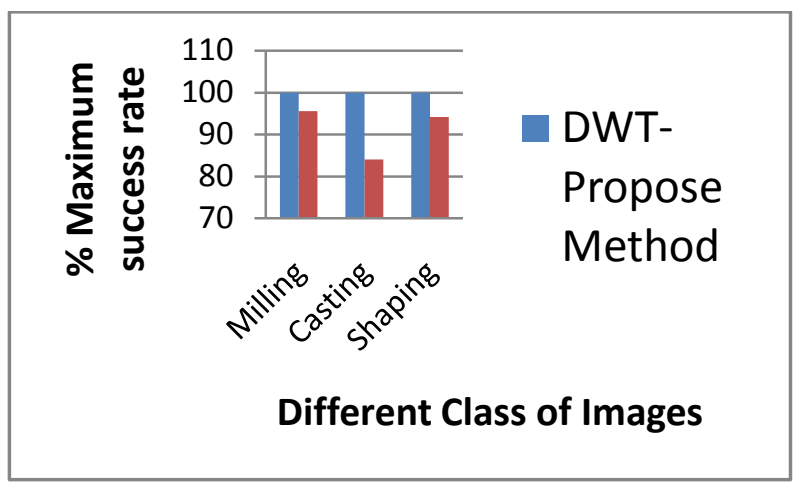

\section{CONCLUSION}

The advantages of the present approach are the non-contact measurements and ease of automation. This work has described the use of machine vision techniques to inspect the surface roughness of a work piece under various milling, casting and shaping operations.

In this study, Wavelet Statistical Features are tested using fuzzy logic classifier for classification of texture images. The performance is measured in terms of Success Rate. The best average success rate is given by energy and standard deviation feature. We got zero success rate if use Standard Deviation only. Milling class gives best success rate as compared to casting and shaping with minimum no of features. 
It is important to note that the rate of correct classification differs with respect to the database; that is the machining process used to manufacture the surface.

The work can be further extended to test the performance for the textures manufactured by other machining processes namely grinding, grit blasting, hand filing, limishing, shot blasting etc.

\section{REFERENCES}

[1] Hon-Son Don, King-Sun Fu, C. R. Liu and Wei-Chung Lin, "Metal Surface Inspection Using Image Processing Techniques", IEEE Transactions of System, Man and Cybernetics, Vol. SMC-14, No.1 January 1984. .

[2] R.T. Chin and C.A. Harlow, "Automated Visual Inspection : A Survey", IEEE Transactions of Pattern Analysis Machine Intelligence, Vol. 4, pp. 557573,November 1982.

[3] G.A. Al-Kindi, R.M. Baul, K.F. Gill. . An application of machine vision in the automated inspection of engineering surfaces, International Journal of Production Research 30(2): 241-253, 1992.

[4] F. Luk, V. Hyunh, and W. North. 1989. Measurement of surface roughness by a machine vision system, Journal of Physics E Scientific Instruments 22: 977-980, 1989.

[5] Irem Y. Tumer, R.S. Srinivasan, Kristin L. Wood, “ characteristic measure for the Representation of Manufactured surface Quality", ASME design Engineering Technology Conference and Design for Manufacturing Conference, Irvine, California, August (18-22) 1996.

[6] S.Livens, P.Scheunders, G.Van de Wouwer, D.Van Dyck, "Wavelets for Texture Analysis", University of Antwerp, Belgium, 30th June 1997.

[7] Wong, B. K., Elliott,M. P. and Rapley, C.W. Automatic casting surface defect recognition and classification. In IEEE Colloquium on Application of Machine Vision , pp. 10/1-10/5, 1995.
[8] V. Niola, G. Nasti and G. Quaremba. A problem of emphasizing features of a surface roughness by means the Discrete Wavelet Transform. $13^{\text {th }}$ International conference on achievements in Mechanical and Material Engineering. $16^{\text {th }}-19^{\text {th }}$ May 2005

[9] W. Zeng, X. Jiang, and P. Scott, "Metrological characteristics of dualtree complex wavelet transform for surface analysis," Meas. Sci.Technol., 16, pp. 1410-1417, 2005.

[10] Smriti H. Bhandari, S. M. Deshpande, "Feature Extraction for Surface Classification -An approach with wavelets",International journal of Computer and Information Science and Engineering, 2007.

[11] R.O. Duda, P.E.Hart, D.G. Stork, Pattern Classification, John Wiley and Sons, Second Edition, 2006.

[12] E.Avci, An expert system based on Wavelet Neural Network-Adaptive Norm Entropy for scale invariant texture classification, Experts Systems with Applications, , vol.32, pp.919-926, Elsevier, 2007.

[13] I. Turkoglu and E.Avci, Comparison of wavelet-SVM and Wavelet-adaptive network based fuzzy inference system for texture classification, Digital Signal Processing, Vol.18, pp.15-24, Elsevier, 2008.

[14] S.M. Mukane, D.S. Bormane, and S.R. Gengaje, "On Size Invariance Texture Image Retrieval using Fuzzy Logic and Wavelet based Features", International Journal of Applied Engineering Research, 6(6), pp.12971310,2011

[15] M. Kokare, P. K. Biswas, and B. N. Chatterji, Rotation-invariant texture image retrieval using rotated complex wavelet filters, IEEE Trans. on Systems, Man, and Cybernetics-Part B: Cybernetics, Vol.36, No.6, 1273-1282, 2006.

[16] P.M. Pawar and R. Ganguli, Genetic fuzzy system for damage detection in beams and helicopter rotor blades,Computer methods in applied mechanics and engineering,Vol.192, 2031-2057, Elsevier 2003. 